\title{
Video Article \\ An Ivor Lewis Esophagectomy Designed to Minimize Anastomotic Complications and Optimize Conduit Function
}

\author{
Neal K. Ramchandani ${ }^{1}$, Kenneth A. Kesler ${ }^{1}$, Jonathon D. Rogers ${ }^{1}$, Nakul Valsangkar ${ }^{1}$, Samatha M. Stokes ${ }^{1}$, Shadia I. Jalal ${ }^{2}$ \\ ${ }^{1}$ Department of Surgery, Division of Cardiothoracic, Indiana University Melvin and Bren Simon Cancer Center \\ ${ }^{2}$ Department of Medicine, Medical Oncology Division, Indiana University Melvin and Bren Simon Cancer Center
}

Correspondence to: Kenneth A. Kesler at kkesler@iupui.edu

URL: https://www.jove.com/video/59255

DOI: doi:10.3791/59255

Keywords: Cancer Research, Issue 158, Esophagectomy, Esophageal Neoplasms, Esophageal Strictures, Quality of Life, Postoperative Complications, and Thoracic Surgery

Date Published: 4/17/2020

Citation: Ramchandani, N.K., Kesler, K.A., Rogers, J.D., Valsangkar, N., Stokes, S.M., Jalal, S.I. An Ivor Lewis Esophagectomy Designed to Minimize Anastomotic Complications and Optimize Conduit Function. J. Vis. Exp. (158), e59255, doi:10.3791/59255 (2020).

\section{Abstract}

We describe a novel esophagogastric anastomotic technique ("side-to-side: staple line-on-staple line", STS) for intrathoracic anastomoses designed to create a large diameter anastomosis while simultaneously maintaining conduit blood supply. This technique aims to minimize the incidence of anastomotic leaks and strictures, which is a frequent source of morbidity and occasional mortality after esophagectomy. We analyze the results of this STS technique on 368 patients and compared outcomes to 112 patients who underwent esophagogastric anastomoses using an end-to-end stapler (EEA) over an 8-year time interval at our institution.

The STS technique involves aligning the remaining intrathoracic esophagus over the tip of the lesser curve staple line of a stomach tube, created as a replacement conduit for the esophagus. A linear stapling device cuts through and restaples the conduit staple line to the lateral wall of the esophagus in a side-to-side fashion. The open common lumen is then closed in two layers of sutures.

There was a total of $12(3.8 \%)$ anastomotic leaks in patients who underwent STS esophagogastric anastomosis. Two of eight patients (25\%) had anastomotic leaks after esophagectomy for end-stage achalasia as compared to a $2.8 \%$ leak rate (10/336) after esophagectomy for other conditions. Eighteen (5.2\%) patients required a median of 2 dilatations for anastomotic stricture after STS anastomosis. Supplemental jejunostomy feedings were required in only $11.1 \%$ of patients undergoing STS anastomoses following hospital discharge. In contrast, patients undergoing EEA anastomoses demonstrated anastomotic leak and stricture rates of $16.1 \%$ and $14.3 \%$ respectively $(p<0.01)$. Time analysis of postoperative contrast studies following the STS technique typically demonstrated a straight/uniform diameter conduit with essentially complete contrast emptying into the small bowel within 3 minutes in $88.4 \%$ of patients.

The incidence of esophagogastric anastomotic leaks and strictures were extremely low using this novel anastomotic technique. Additionally we believe that based on time and qualitative analyses of postoperative contrast studies, this technique appears to optimize postoperative upper gastrointestinal tract function; however, further comparative studies are needed.

\section{Video Link}

The video component of this article can be found at https://www.jove.com/video/59255/

\section{Introduction}

Esophagogastric anastomotic leaks represent a not uncommon complication after esophagectomy ${ }^{1}$. Additionally, an anastomotic leak has been associated to unfavorable longer-term outcomes including hospital readmission, early mortality, and occasionally poor quality of life ${ }^{2,3,4}$. An anastomotic stricture represents a long-term complication, which can also be a consequence of an anastomotic leak ${ }^{5}$. Anastomotic strictures both negatively impact quality of life as well as escalate the cost of care.

As more esophageal cancer patients are living longer as a result of endoscopic detection of early stage adenocarcinoma and induction chemoradiation therapy for more locally advanced cancers, optimizing stomach conduit function also becomes important. Gastric conduit "function" mainly, however, relies on gravity for drainage. Gastric conduit construction and orientation can affect upper gastrointestinal tract function and therefore poor conduit "function" can be a result of technical issues.

We have used a novel "side-to-side: staple line-on-staple line" (STS) technique for intrathoracic esophagogastric anastomosis since 2009. This technique is designed to create a larger diameter anastomosis as compared to anastomoses made with end-to-end staplers (EEA) while simultaneously maintaining conduit blood supply to reduce the incidence of anastomotic leaks and strictures. We describe this novel anastomotic technique and additionally described gastric conduit construction and orientation to optimize function. We compared the results of this STS technique to anastomoses performed with EEA staplers over an 8-year time interval at our institution. 


\section{Protocol}

This study was approved by Indiana University-Purdue University in Indianapolis institutional review board (1109006832). This procedure was performed on all patients requiring resection and resection of the distal intrathoracic esophagus for malignant or benign diseases since 2009 at Indiana University Hospital.

\section{Pre-anesthesia phase}

1. Place an epidural catheter.

2. Obtain standard central venous and radial arterial access.

3. Anesthetize and intubate using a double lumen left-sided endobronchial tube for selective lung ventilation during the thoracic phase of the operation.

\section{Initial laparotomy phase}

1. Perform an upper midline laparotomy including excision of the zyphoid process with electrocautery. Place a self-retaining abdominal wall retractor for peritoneal cavity exposure.

2. Perform a wide Kocher maneuver, which mobilizes the entire duodenum from the retroperitoneum. This maneuver not only straightens the gastric conduit, but also allows well-vascularized portions of the stomach conduit to be advanced above the azygos arch.

3. Temporarily place a standard size laparotomy sponge under the duodenum to elevate the conduit, which is removed in the final laparotomy phase.

4. Divide the gastrocolic ligament by cauterizing and dividing the omental blood vessels with a standard energy device. Avoid manipulation of the right gastroepiploic vessels by placing a nasogastric tube along the greater curvature, which is used as a "handle" retracting the stomach upward.

5. Score the peritoneum circumferentially around the diaphragmatic crus with electrocautery and temporarily place a Penrose drain around the intraabdominal esophagus.

6. Ligate the left gastroepiploic and short gastric vessels with a combination of suture and surgical clips. Then divide.

7. In cases of malignancy, mobilize the celiac lymph bearing tissues from the superior aspect of the pancreas, which includes ligation and division of both left gastric artery and vein.

8. Clear the lesser gastric curve of fat and blood vessels typically 3 to $5 \mathrm{~cm}$ distal to the gastroesophageal junction with vascular endostaplers. In cases of malignancy, plan the operations to achieve tumor free 3 to $5 \mathrm{~cm}$ distal stomach and 5 to $7 \mathrm{~cm}$ proximal esophageal surgical margins. As tumor locations range from the mid-esophagus to the gastric cardia, achieving adequate distal stomach and proximal esophagea margins results in conduits of varying lengths and associated lower or higher intrathoracic esophagogastric anastomoses. The specific site of the lesser curve clearing is therefore somewhat variable and dependent on exact tumor location.

9. Cleave the upper gastric fundus and cardia from the remainder of the stomach typically using anywhere from 3 to $560 \mathrm{~mm}$ endoscopic staplers beginning from the greater gastric curve into the cleared area on the lesser gastric curve.

10. Gastric conduit creation

1. Debulk lesser omental fat around the right gastric vessels, which allows the conduit to be straightened as well as lengthened. Division of right gastric vessels as they insert onto the lesser curve at the incisura will provide further lengthening.

2. Secure the stomach at three points and provide outward retraction (Figure 1).

3. Create a narrow uniform diameter conduit (average $7-8 \mathrm{~cm}$ ) with an initial fire of the $100 \mathrm{~mm}$ stapler, which delivers two rows of $4.8 \mathrm{~mm}$ staples aiming just beneath the previous staple line. Completion of the conduit construction usually requires 2 or 3 additional fires of the $60 \mathrm{~mm}$ endoscopic tri-stapler into the cleared area on the lesser gastric curve. In cases of malignancy, send the stomach defect as the "distal stomach margin" for frozen section pathologic analysis.

4. Inspect the tip of the conduit. Within 3 to $5 \mathrm{~min}$, punctate bright red bleeding is usually seen through the lesser curve staple line representing good conduit perfusion. If no punctate bright red oozing is present, slightly trim the conduit tip with another $100 \mathrm{~mm}$ stapler until punctate bleeding is seen. Tri-staplers are not used for conduit trimming as cutting through and restapling the conduit tip during the intrathoracic anastomosis may be difficult.

5. Perform a standard Heineke-Mikulicz pyloroplasty in order to assure good conduit emptying.

6. Initially estimate how high in the chest the conduit will reach by comfortably stretching the conduit towards the neck without undue tension.

7. Open the right pleura through the diaphragmatic crus. Place the conduit tip into the right chest keeping the lesser curve staple line facing rightward.

8. Temporarily close the abdomen with a few interrupted fascial sutures and skin staples.

\section{Thoracic phase}

1. Place the patient in the left lateral decubitus position.

2. Perform a serratus muscle sparing right thoracotomy through the $5^{\text {th }}$ intercostal space. Divide the intercostal muscle underneath the incision within 3 to $5 \mathrm{~cm}$ of the vertebral body posteriorly and the sternum anteriorly, allowing additional movement of the $5^{\text {th }}$ and $6^{\text {th }}$ ribs with minimal risk of fracture or bruising.

3. Excise the arch of the azygos vein and divide the inferior pulmonary ligament. In cases of malignancy, perform en bloc dissection by mobilizing the distal two-thirds of the intrathoracic esophagus with all surrounding soft tissues from the pericardium anteriorly to the aorta posteriorly esophageal blood vessels. Carefully occlude lymphatics with surgical clips before division during this dissection. 
4. Deliver the conduit into the right chest until there is no redundancy, limiting tension on the right gastric and right gastroepiploic vascular pedicles to determine the superior extent of esophageal dissection needed.

5. At the level of the tracheal carina, dissect the esophageal wall from surrounding mediastinal soft tissues superiorly to a level where the tip of the conduit reaches without tension, which is usually 3 to $5 \mathrm{~cm}$ superior to the carina. Again however, given varying locations of tumors and length of stomach conduits created during the abdominal phase, establish anastomoses lower near the carina for gastric cardia tumors and higher at the thoracic inlet for tumors involving the middle intrathoracic esophagus.

6. Creation of the esophagogastric anastomosis

1. Align the mid left lateral aspect of the upper third of the intrathoracic esophagus over the lesser curve staple line.

2. Place four tacking sutures approximately 2 to $3 \mathrm{~cm}$ apart in order to maintain alignment (Figure 2).

3. Transect the esophagus $1 \mathrm{~cm}$ distal to the inferior set of tacking sutures. In cases of malignancy, send a ring of esophagus at this level as the "proximal esophageal margin" for frozen section pathologic examination prior to proceeding.

4. Create a corresponding 1 to $2 \mathrm{~cm}$ opening in the conduit across the lesser curve staple line.

5. Place interrupted sutures within the common lumen, incorporating the adjacent conduit and esophageal walls beginning in the middle through the lesser curve staple line and progressing to either side (Figure 3)

6. Place the narrow anvil of a $45 \mathrm{~mm}$ endoscopic stapler with a $4.1 \mathrm{~mm}$ staple height in the esophageal lumen and the large anvil in the conduit. To optimize the length of the side-to-side communication between the esophagus and the conduit, trim $5 \mathrm{~mm}$ from distal plastic tip of stapler cartridge with an oscillating saw.

7. Fire the stapler, which cuts through and restaples the lesser curve staple line (Figure 4). The length of the cut end of the esophagus is usually somewhat longer than the edge of the gastric conduit, so remove an ellipse of the conduit over the lesser curve staple line rather than extending the rent laterally, preserving collateral blood supply (Figure 4 inset).

8. Close the open common lumen in two layers of sutures beginning with an inner layer of inverted interrupted 3-0 polyglactin suture followed by a second layer of interrupted 3-0 silk using a Lembert technique (Figure 5).

9. Over sew the upper aspect of the lesser curve conduit staple line with interrupted 3-0 silk sutures in a Lembert fashion extending inferiorly, until the right gastric vessels are encountered.

10. Have the anesthesiologist place a nasogastric tube into the conduit to the level of the crus by palpation.

11. For anastomoses created near the thoracic inlet, use a pleural flap to seal the anastomosis in the posterior mediastinum. For anastomoses in the middle aspect of the posterior mediastinum, mobilize a pericardial fat pat from anterior mediastinum and loosely wrapped to cover esophagogastric anastomosis (Figure 6 and Figure 7).

12. Insert and position two 28-French chest tubes within the right hemithorax, one anterior and the other posterior, next to, but not abutting the conduit. Close the thoracotomy incision.

\section{Re-laparotomy: "double flip" phase}

1. Return patient to the supine position and reopen the midline laparotomy incision.

2. Inspect the right gastroepiploic fat and carefully push any excess fat upwards through the left diaphragm crus into the chest.

3. Secure the right gastroepiploic fat and conduit to the diaphragmatic crus with interrupted 2-0 silk sutures (Figure 8). Place a feeding jejunostomy tube in select patients, primarily in elderly patients or patients with preoperative nutritional deficits.

4. Formally close the midline laparotomy incision.

5. Keep patients intubated the evening of surgery with extubation planned the following morning

6. Provide pain control with epidural catheters placed just prior to anesthetic induction for the first 3 to 4 days, which is supplemented by intravenous narcotics.

7. Obtain contrast upper gastrointestinal series 5 to 7 days postoperatively to assess for anastomotic integrity.

8. If no anastomotic leak is identified, remove the nasogastric tube.

9. Instruct patients to advance to a regular diet in 2 to 3 weeks after discharge as tolerated.

\section{Representative Results}

From 2009 to 2017, a total of 368 patients were identified who underwent an STS intrathoracic esophagogastric anastomosis and of these 12 $(3.8 \%)$ had anastomotic leaks. Five of these patients demonstrated grade I/II leaks and required no intervention. Six patients and one patient respectively experienced grade III and grade IV leaks requiring endoscopic stenting and/or surgical intervention ${ }^{2}$. A leak rate of $25 \%(2 / 8)$ was observed after esophagectomy for end-stage achalasia as compared to a $2.8 \%$ leak rate (10/336) where esophagectomy was performed for other conditions. There were $4(1.1 \%)$ patient who died postoperatively, none of which had an anastomotic complication. Of STS patients, $18(5.0 \%)$ required a median of 2 dilatations for symptomatic anastomotic strictures. Supplemental jejunostomy feedings were required in only $11.1 \%$ of these patients following hospital discharge. In contrast, of the 112 patients identified who underwent thoracoscopic end-to-end mechanical stapler (EEA) anastomosis over this same time interval, $16.1 \%$ and $14.3 \%$ demonstrated anastomotic leaks and symptomatic strictures respectively $(p<0.01)$ despite all $(100 \%)$ of these patients being maintained on a limited diet with supplemental jejunostomy tube feedings for at least one month following surgery (Table 1). Demographic and comorbidities of both STS and EEA groups were statistically similar; however, there was a trend towards more cardiac disease in the STS cohort (Table 2). Time analysis of postoperative contrast studies in first 208 patients who underwent an STS anastomosis typically demonstrated a straight/uniform diameter conduit with essentially complete $(>95 \%)$ contrast emptying into the small bowel within 3 min in $184(88.4 \%)$ patients. The remaining $11.6 \%$ of patients studied, had near complete $(>95 \%)$ contrast emptying into the small bowel within 5 minutes. No patient studied had contrast transit time in excess of 5 minutes. 


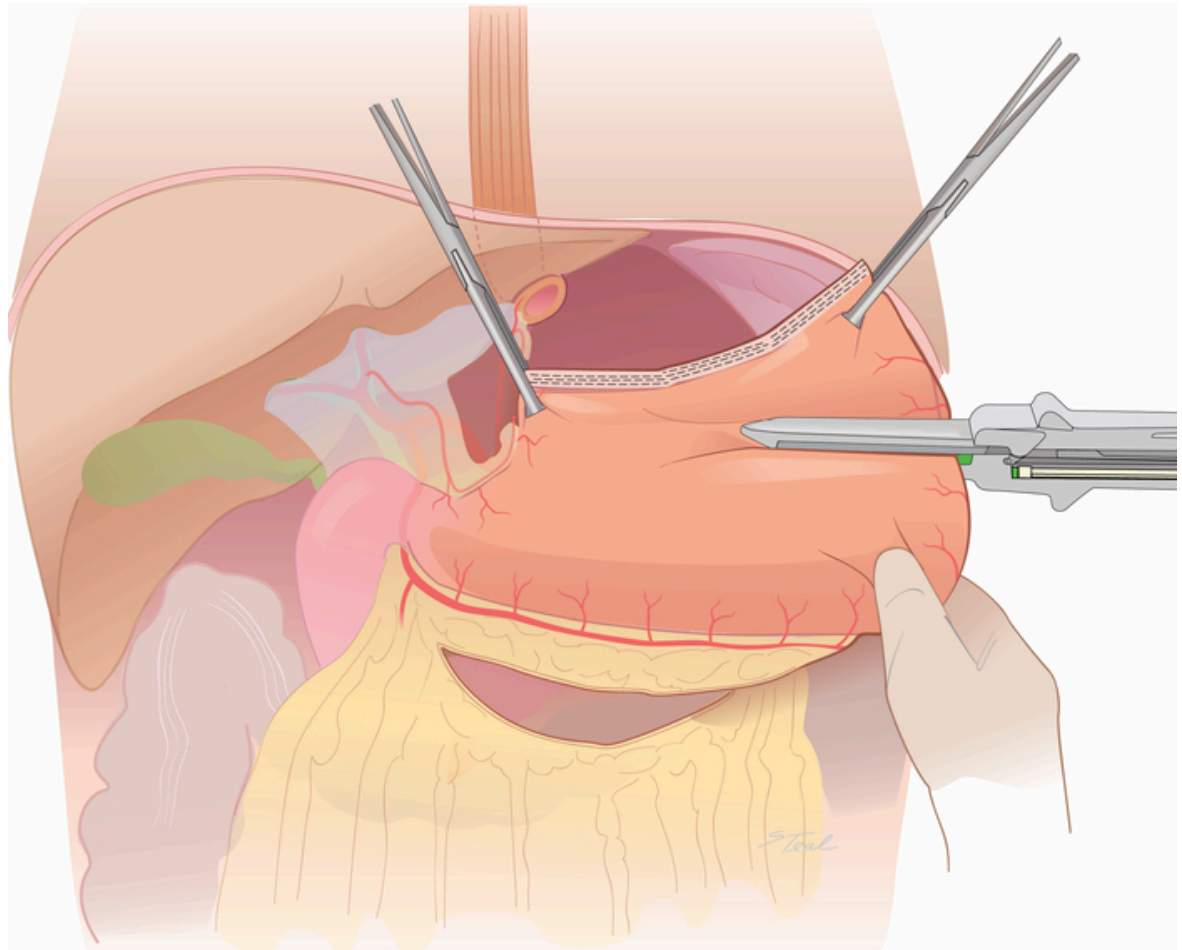

Figure 1: Creation of the stomach conduit. The stomach is secured at three points, which are retracted outward. A relatively uniform diameter conduit is created with staplers aiming just inferior to the previous staple line on the lesser curve. (Taken from with permission ${ }^{11}$.) Please click here to view a larger version of this figure.

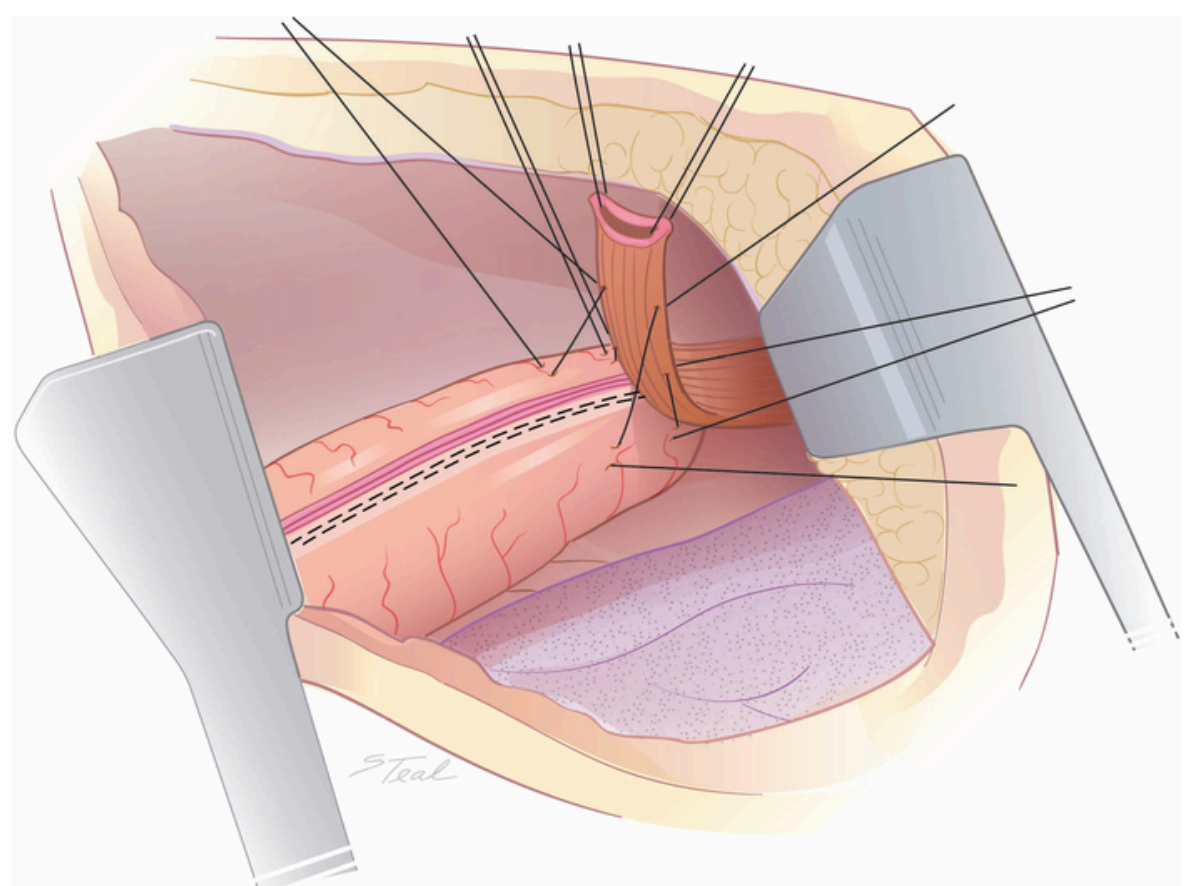

Figure 2: Alignment of the esophagus over the stomach conduit. The mid left lateral aspect of the upper third of the intrathoracic esophagus is aligned over the lesser curve staple line at the tip of the conduit with 4 initial tacking sutures approximately 2 to $3 \mathrm{~cm}$ apart. (Taken from with permission ${ }^{11}$.) Please click here to view a larger version of this figure. 


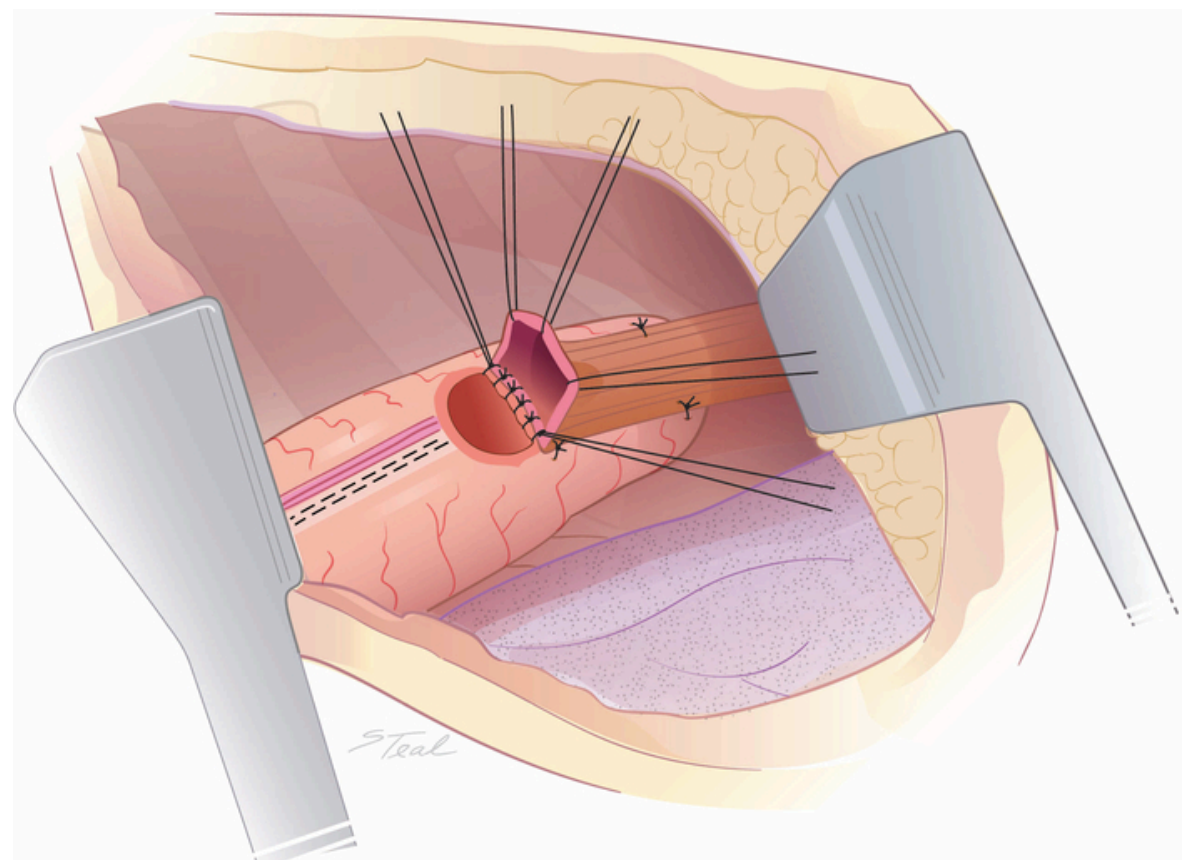

Figure 3: Preparation for a side-to-side communication between the esophagus and stomach conduit. A 1 to $2 \mathrm{~cm}$ rent is made in the stomach conduit across the lesser curve staple line. Tacking sutures are placed between the adjacent conduit and esophageal walls beginning in the middle through the lesser curve staple line then two sutures on either side. (Taken from with permission ${ }^{11}$.) Please click here to view a larger version of this figure.

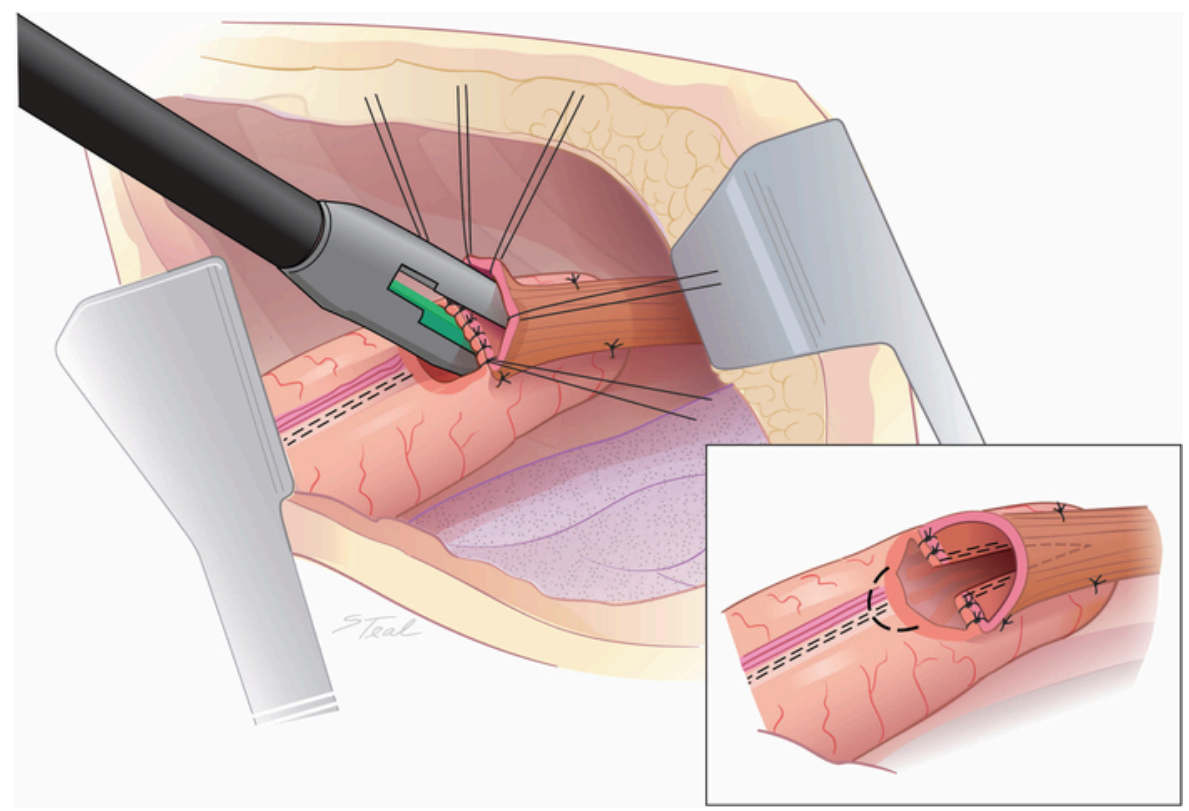

Figure 4: Creation of a side-to-side communication between the esophagus and stomach conduit. A $45 \mathrm{~mm}$ endoscopic stapler is fired cutting through and restapling the lesser curve staple line. Typically only $2 / 3$ rds of the stapler length is used. An ellipse of conduit is removed over the lesser curve staple line (dotted line) rather than extending the rent laterally to equalize the length of the cut end of the esophagus and gastrotomy. (inset) (Taken from with permission ${ }^{11}$.) Please click here to view a larger version of this figure. 


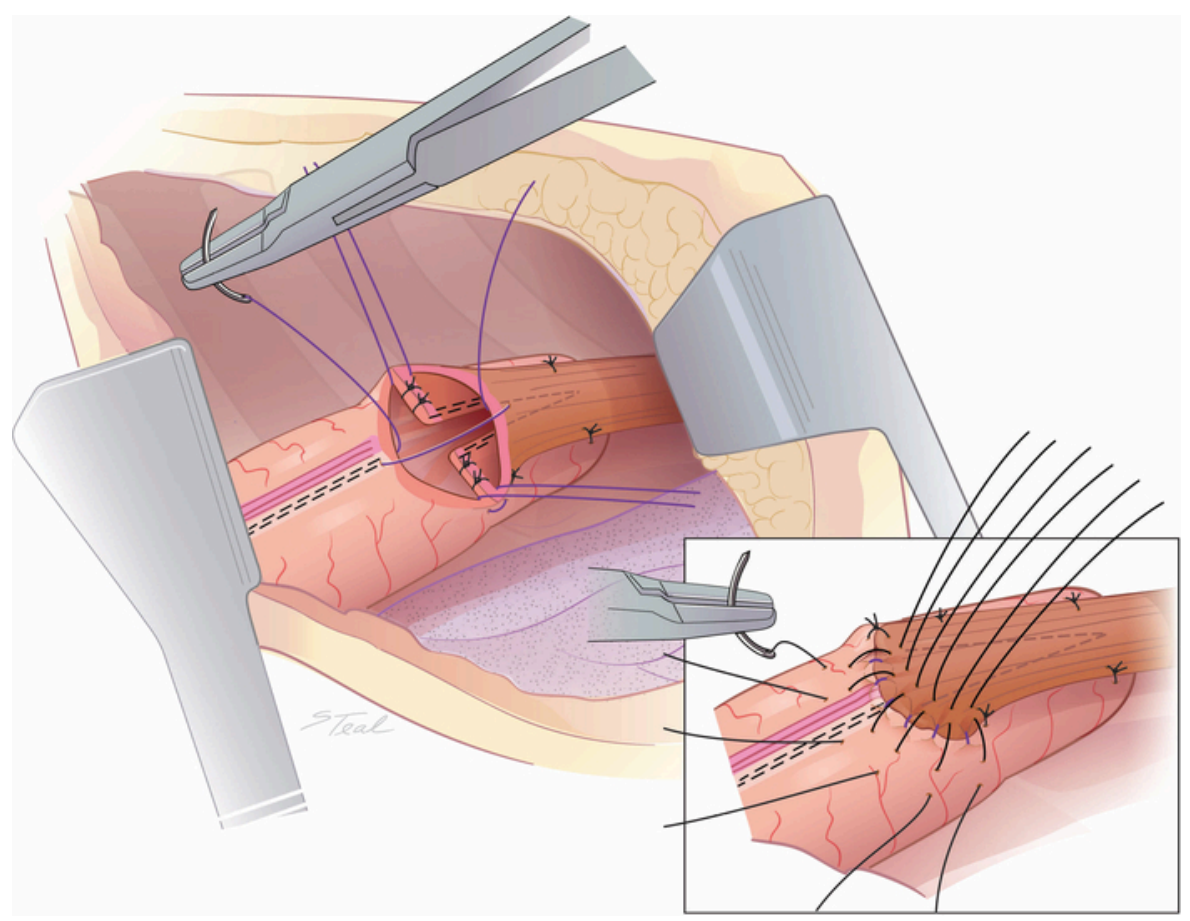

Figure 5: Closure of the open common lumen. The open common lumen is hand-closed in two layers of sutures beginning with inverted interrupted absorbable sutures. The first suture layer is imbricated by a second layer of Lembert silk sutures placing the stomach suture a few $\mathrm{mm}$ inferior to the first layer of sutures. (inset) (Taken from with permission ${ }^{11}$.) Please click here to view a larger version of this figure.

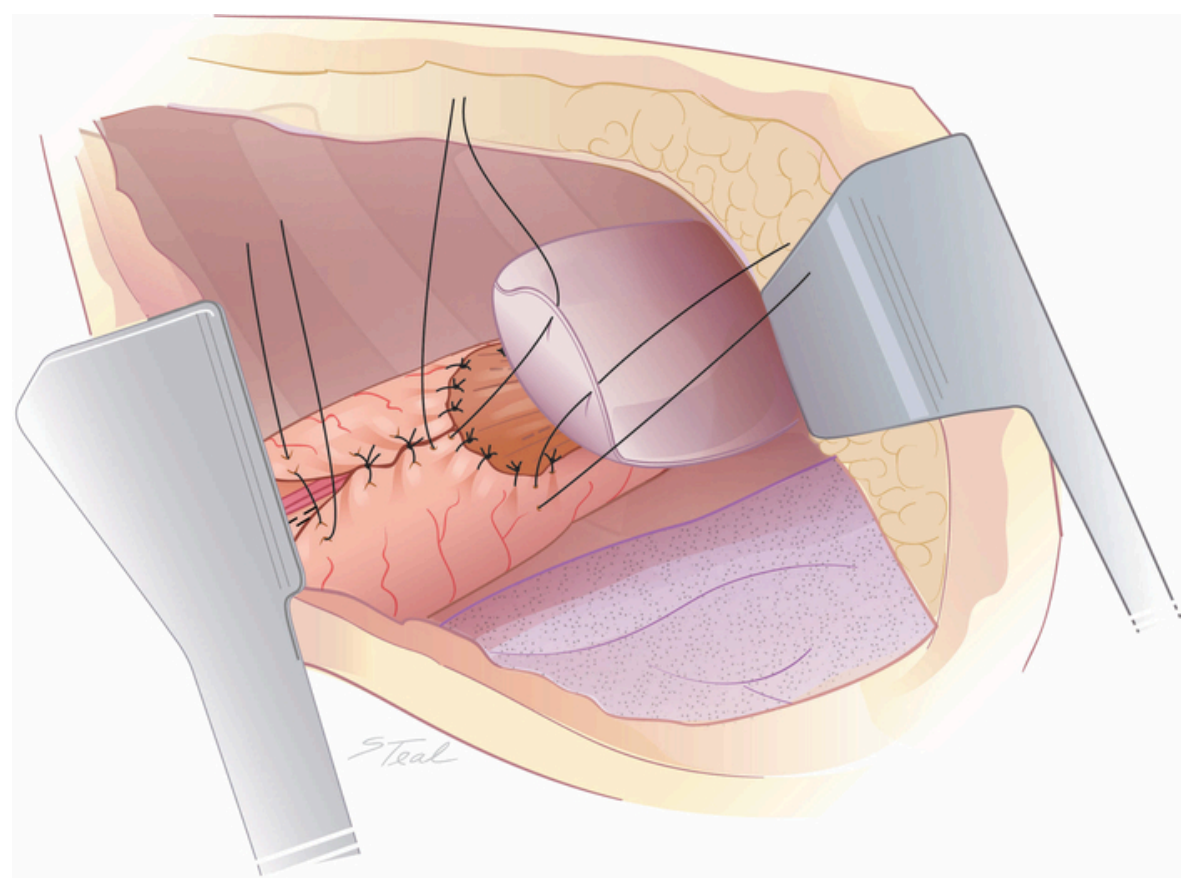

Figure 6: Over sew of the lesser curve staple line and pleural buttress of the anastomosis. The superior aspect of the lesser curve conduit staple line can be over sewn at this point with interrupted silk sutures inferiorly until the right gastric vessels are encountered. For anastomoses established in near the thoracic inlet, a flap of mobilized pleura is tacked to the stomach conduit to contain small anastomotic leaks. (Taken from with permission ${ }^{11}$.) Please click here to view a larger version of this figure. 


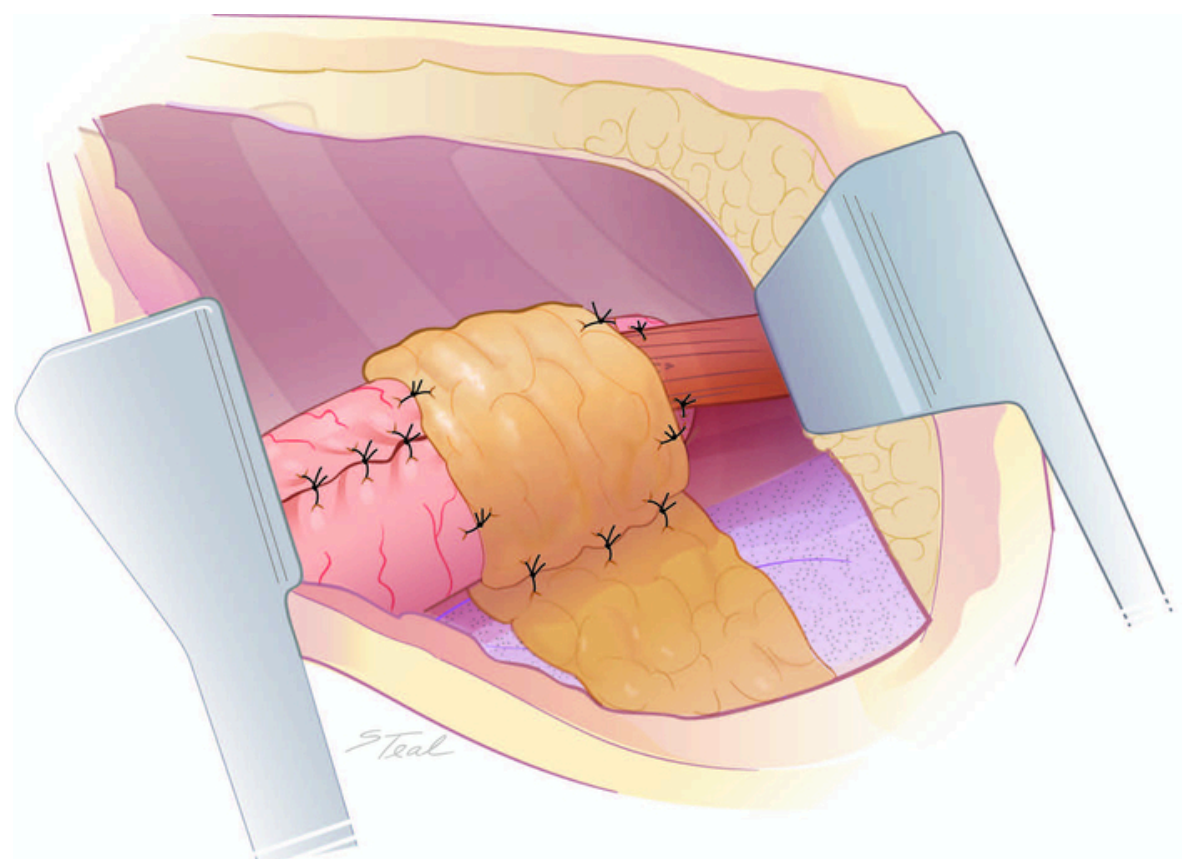

Figure 7: Vascularized soft tissue buttress of the anastomosis. Pericardial fat is loosely wrapped around anastomoses created in the middle aspect of the posterior mediastinum to contain any small areas of anastomotic dehiscence. Please click here to view a larger version of this figure. 


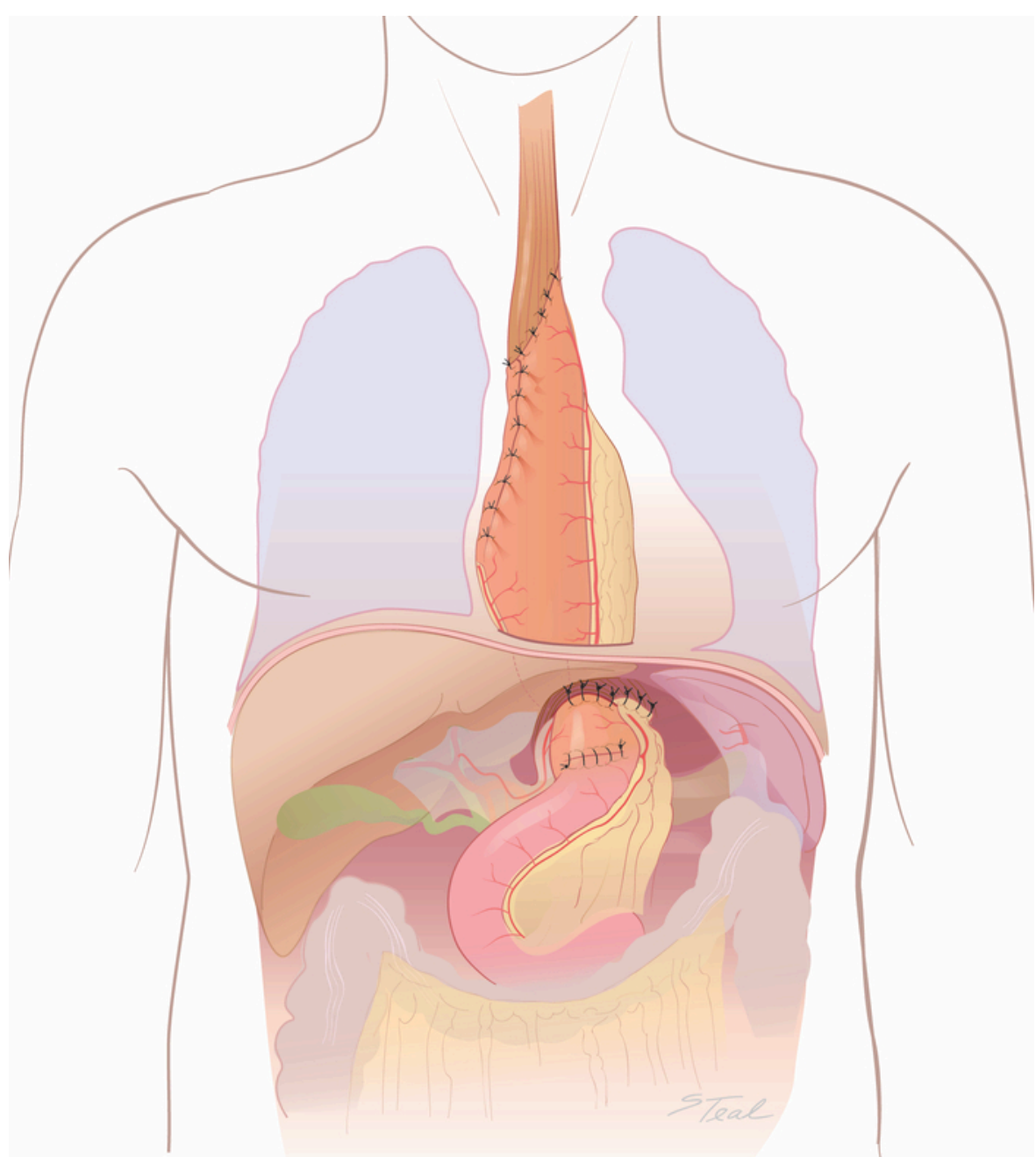

Figure 8: Final (“double flip") laparotomy phase. The laparotomy incision is reopened. The right gastroepiploic fat and conduit carefully tacked to the crus with 2-0 silk sutures. (Taken from with permission ${ }^{11}$.) Please click here to view a larger version of this figure.

\begin{tabular}{|l|l|l|}
\hline & STS (N=368) & EEA (N=112) \\
\hline Anastomotic Leaks & $3.8 \%^{*}$ & $16.1 \%$ \\
\hline Anastomotic Stricture & $5.2 \%^{*}$ & $14.3 \%$ \\
\hline Post Op J-Tube Usage & $11.0 \%^{*}$ & $100.0 \%$ \\
\hline
\end{tabular}

Table 1. Anastomotic leaks/strictures and use of postoperative feeding jejunostomy (Post Op J-Tube Usage) following hospital discharge comparing patients undergoing open STS and thoracoscopic EEA esophagogastric anastomoses using an Ivor Lewis approach performed at Indiana University Simon Cancer Center from 2009 to 2017. ( ${ }^{*} p$ value $<0.01$, chi-square) 


\begin{tabular}{|l|l|l|l|}
\hline \multicolumn{2}{|l|}{ STS (n=278) } & EEA (n=82) & \multicolumn{2}{l|}{ P-Value } \\
\hline Age at Diagnosis (yrs) & $60.3 \pm 11.4(23-84)$ & $60.6 \pm 9.0(38-80)$ & 0.80 \\
\hline Gender & $228(82.0 \%)$ & $69(84.1 \%)$ & 0.66 \\
\hline Male & $50(18.0 \%)$ & $13(15.9 \%)$ & \\
\hline Female & $114(41.0 \%)$ & $24(29.3 \%)$ & 0.06 \\
\hline Cormorbidity & $70(25.2 \%)$ & $19(23.2 \%)$ & 0.71 \\
\hline Cardiac & $32(11.5 \%)$ & $12(14.6 \%$ & 0.45 \\
\hline Diabetes Mellitus & \multicolumn{3}{l|}{} \\
\hline COPD & $237(85.3 \%)$ & $74(90.2 \%)$ & 0.25 \\
\hline Histology & $41(14.7 \%)$ & $8(9.8 \%)$ & \\
\hline Adenocarcinoma & $200(71.9 \%)$ & $59(71.9 \%)$ & 0.99 \\
\hline Other Diagnoses &
\end{tabular}

Table 2: Demographic and comorbidity comparison open STS and thoracoscopic EEA anastomoses using an Ivor Lewis approach performed at Indiana University Simon Cancer Center from 2009 to 2015. Mean and standard deviation with range given for continuous variables. $P$ values for continuous variables generated by Student's t-test and chi-square for discrete variables.

\section{Discussion}

Esophagectomy represents a very extensive surgical procedure. Adverse long-term quality of life has been linked to patients experiencing postoperative complications including anastomotic leaks ${ }^{3}$. Risk factors for an anastomotic leak primarily include creation of an anastomosis with poor blood supply. An anastomotic leak not only represents a significant source of postoperative morbidity, but also can commonly result in stricture. A stricture can also be a result of technical issues including performing a small diameter anastomosis. Besides impacting quality of life, strictures add to overall medical costs when dilatation is needed ${ }^{5}$. Accurately performing several steps is of utmost importance to minimize complications as well as achieve good oncologic and upper gastrointestinal tract functional outcomes.

Esophagogastric anastomosis can be accomplished by several methods, including hand sewn, EEA, and linear stapler techniques representing the majority. A report from the Society of Thoracic Surgeons General Thoracic Database cited an overall leak rate of $9.3 \%$ in patients undergoing intrathoracic esophagogastric anastomoses ${ }^{6}$. While postoperative mortality due to anastomotic leak seems to be decreasing, subsequent stricture rates remain high, ranging between 10 and $56 \%{ }^{7}$. Collard and Orringer described a linear stapler technique to create a side-to-side cervical esophagogastric anastomosis ${ }^{8,9}$. The posterior triangulated opening formed by the linear stapler was demonstrated to result in a low leak rate as well as resistance to stricture. A retrospective study from Mayo Clinic reported a $5.6 \%$ incident of intrathoracic anastomotic leak in 177 patients where a linear stapled technique was used versus an $8.3 \%$ leak rate in 48 patients who underwent an EEA stapled anastomosis. While this difference did not reach statistical difference, the one-year probability for stricture was $32 \%$ after EEA anastomosis as compared to only $8.6 \%$ with linear stapled techniques, which was significant ${ }^{5}$. Wang and colleagues performed a prospective clinical trial involving 155 patients who were randomized into one of three esophagogastric anastomotic methods ${ }^{10}$. Impressively, no postoperative strictures developed in patients undergoing a linear stapler technique as compared to $9.6 \%$ and $19.1 \%$ in the hand sewn and circular stapled cases respectively, which was statistically significant. Prior studies involving linear stapler anastomoses have utilized the anterior wall of the stomach conduit for the anastomotic site. This approach may lead to an ischemic strip of conduit between the lesser curve staple line and the anastomosis predisposing to leak. Novel to our technique, collateral blood supply to the stomach conduit tip is preserved by cutting through and restapling the lesser curve staple line.

There are limitations to the study. First, this represents a retrospective analysis. Despite the retrospective nature however, we utilized this STS approach uniformly in all patients undergoing surgery for mid-esophageal to proximal stomach pathology over the study interval as an "intent to treat" including stable patients who sustained esophageal perforations during dilatation for stricture where repair was not possible. Common to any intrathoracic side-to-side anastomotic technique is the need to dissect an additional 3 to $4 \mathrm{~cm}$ of proximal esophagus, potentially decreasing the length of surgical esophageal margin in cases of malignancy and also potentially resulting in some degree of esophageal devascularization. To avoid devascularization, a critical point is not to dissect the intrathoracic esophagus any further superiorly toward the neck than the tip of the stomach conduit will reach without tension.We speculate the higher leak rate observed using this technique for patients with end-stage achalasia may be related to further devascularization of a thicken esophageal wall after mobilization to perform an STS anastomosis where the preexisting blood supply may be poor. Esophageal dilatation frequently seen in achalasia cases makes hand sewing the open common lumen very difficult, which may also be a factor.Based on this experience, we now believe that achalasia is a contraindication to perform STS intrathoracic anastomoses. Of note, for long or more central cancers where an esophagogastric anastomosis needs to be created near the thoracic inlet to achieve an adequate proximal esophageal margin, we have utilized a somewhat shorter initial side-to-side communication not utilizing the entire length of the $45 \mathrm{~mm}$ GIA which however has potential to be more prone to stricture formation.

Unlike the esophagus, the stomach is a passive conduit, gravity dependent for drainage. Several variables including conduit diameter and length as well as conduit orientation can, therefore, significantly impact upper gastrointestinal tract function. Ingested food has the potential to hang up in three locations: the esophagogastric anastomosis, the stomach body, and gastric outlet. Poor conduit function can be a result of technical issues in any of these three areas. Poor conduit emptying paradoxically can cause more "reflux," not only negatively impacting quality of life but also occasionally resulting in aspiration. "Minimally invasive" (laparoscopic/thoracoscopic) approaches, which utilize an EEA stapler for esophagogastric anastomoses, although still representing the minority of esophagectomy cases performed, have become increasingly 
popular. We believe however our open technique as described not only allows creations of precise STS esophagogastric anastomosis to reduce the stricture rates but additionally optimizes conduit construction and orientation with a straight non-redundant stomach conduit including pyloroplasty with minimal tendency for ingested food materials to hang up in these areas as compared to thoracoscopic approaches. The measured contrast transit times from mouth to small bowel on routine postoperative studies would support excellent conduit function with our technique, however comparative studies using other techniques are needed. Our observations would support minimal and self-limiting "dumping" symptoms in the vast majority of STS patients however specific quality of life assessments are currently underway. Finally, with the thoracotomy approach described, we have observed little difference with respect to acute and long-term postoperative discomfort as compared to patients undergoing a thoracoscopic approach at our institution.

In summary, we believe this novel STS technique can significantly reduce the morbidity and occasional mortality of esophagogastric anastomotic complications following esophagectomy. Conduit construction and orientation as described additionally optimizes upper gastrointestinal tract function. Finally, this technique is easily adapted and reproducible.

\section{Disclosures}

The authors have no disclosures.

\section{Acknowledgments}

None.

\section{References}

1. Cassivi, S.D. Leaks, strictures, and necrosis: a review of anastomotic complications following esophagectomy. Seminars in Thoracic and Cardiovascular Surgery. 16, 124-32 (2004).

2. Low, D.E. et al. International consensus on standardization of data collection for complications associated with esophagectomy: Esophagectomy complication consensus group (ECCG). Annals of Surgery. 262, 286-94 (2015).

3. Derogar, M., Orsini, N., Sadr-Azodi, O., Langergren P. Influence of major postoperative complications on health-related quality of life among long-term survivors of esophageal cancer surgery. Journal of Clinical Oncology. 30,1615-9 (2012).

4. Kofoed, S.C. et al. Intrathoracic anastomotic leakage after gastroesophageal cancer resection is associated with increased risk of recurrence. Journal of Thoracic and Cardiovascular Surgery. 150, 42-8 (2015).

5. Price, T.N. et al. A comprehensive review of anastomotic technique in 432 Esophagectomies. Annals of Thoracic Surgery. 95, 1154-60 (2013).

6. Kassis, E.S. et al. Predictors of anastomotic leak after esophagectomy: an analysis of the Society of Thoracic Surgeons General Thoracic Database. Annals of Thoracic Surgery. 96, 1919-26 (2013).

7. Yuan, Y., Wang, K.N., Chen, L.Q. Esophageal anastomosis. Diseases of the Esophagus. 28, 127-37 (2015).

8. Collard, J.M., Romagnoli, R., Goncette, L., Otte, J.B., Kestens P.J. Terminalized semimechanical side-to-side suture technique for cervical esophagogastrostomy. Annals of Thoracic Surgery. 65, 814-7 (1998).

9. Orringer, M.B., Marshall, B., lannectoni M.D. Eliminated the cervical esophagogastric anastomotic leak with a side-to-side stapled anastomosis. Journal of Thoracic and Cardiovascular Surgery. 119, 277-88 (2000).

10. Wang, W.P., Gao, Q., Wang, K.N., Shi H, Chen L.Q. A prospective randomized controlled trial of semi-mechanical versus hand-sewn or circular stapled esophagogastrostomy for prevention of anastomotic stricture. World Journal of Surgery. 37, 1043-50 (2013).

11. Kesler, K.A. Outcomes of a novel intrathoracic esophagogastric anastomotic technique. The Journal of Thoracic and Cardiovascular Surgery. 156 (4), 1739-1745 (2018). 